\title{
Original
}

\section{The Stromal Cell-derived Factor-1 Expression Protected in Periodontal Tissues Damage during Occlusal Traumatism}

\author{
Kazuko T Goto ${ }^{1)}$, Hiroshi Kajiya ${ }^{2,3)}$, Takashi Tsutsumi ${ }^{4)}$, Munehisa Maeshiba ${ }^{4)}$, Takashi Tsuzuki ${ }^{4)}$, \\ Kimiko Ohgi ${ }^{5)}$, Minoru Kawaguchi' ${ }^{3)}$ Jun Ohno ${ }^{3)}$ and Koji Okabe ${ }^{2)}$ \\ ${ }^{1)}$ Department of Dental Hygiene, Fukuoka College of Health Sciences, Fukuoka, Japan \\ ${ }^{2)}$ Department of Physiological Science and Molecular Biology, Fukuoka Dental College, Fukuoka, Japan \\ ${ }^{3)}$ Research Center for Oral Medicine, Fukuoka Dental College, Fukuoka, Japan \\ ${ }^{4)}$ Department of Oral Rehabilitation, Fukuoka Dental College, Fukuoka, Japan \\ ${ }^{5)}$ Department of Odontology, Fukuoka Dental College, Fukuoka, Japan \\ (Accepted for publication, December 3, 2020)
}

\begin{abstract}
Mechanical stress (MS) during hyperocclusion results in elimination of the alveolar hard line, enhancement of bone resorption, and shedding of a tooth, resulting in trauma of occlusion. Several studies have indicated that MS induces cytokine and chemokine expression during alveolar bone absorption in periodontitis and orthodontic treatment. However, it remains unknown regarding the effect of hyperocclusal MS on maintenance of alveolar bone metabolism. Using in vivo and in vitro hyperocclusion models, we investigated the effect of MS on relationships between the expression of stress-dependent and osteoblastogenesis-associated chemokines. In the in vitro model with 6-weeks old mice, MS upregulated the expression of stromal cell-derived factor-1 (SDF-1) in periodontal tissues, a self-renewal chemokine in bone marrow stem cell day 4 after MS. In contrast, in the in vivo model with 30-weeks old mice, SDF-1 expression was significantly upregulated at day 1 after MS in periodontal tissues. MS induced the expression of SDF-1 in the PDL root branch and of the SDF-1 receptor CXCR4 in the dental pulp and bone marrow on day 4 in an in vivo mice model. MS upregulated the expression of SDF-1 in PDL cells. SDF-1 binds to CXCR4, resulting in MS-resistant alveolar bone protection during occlusal traumatism.
\end{abstract}

Key words: Alveolar bone, Occlusal traumatism, Periodontal tissues, SDF-1

\section{Introduction}

Early contact and lateral rolling of abutment teeth in partial dentures exert intermittent mechanical stress (iMS) on the periodontal tissue. MS with regular occlusions suppresses tooth movement, and excessive MS is associated with disappearance of alveolar bone wiring and expansion of the periodontal ligament (PDL). Further, MS reportedly caused damage to periodontal tissue, including bone resorption and loss of teeth, thereby causing occlusal trauma ${ }^{1-3)}$. The regulatory role of MS in the process of alveolar bone remodeling has been considered crucial $^{4,5)}$.

Chemokines are basic, heparinous, 92-99-amino-acid-long cytokines; they have a low-molecular weight of approximately 8-14 kDa. Approximately 50 types of $\mathrm{N}$-terminal cysteine residual sequences responsible for intramolecular disulfide bonds have been identified. Chemokines are divided into the following four main subfamilies: C, $\mathrm{CC}, \mathrm{CXC}$, and $\mathrm{CX} 3 \mathrm{C}^{6-8)}$. Chemokines play functional roles in the migration and infiltration of leukocytes into tissues in inflammatory and immune reactions as well as in the maintenance of ecological homeostasis and pathology. Stromal cell-derived factor-1 (SDF-1) is a chemokine for the imperative maintenance of hematopoietic stem cells in the bone marrow, and is reportedly involved in the movement of teeth by orthodontic forces and in the migration of blood stem cells. SDF-1 bind into its chemokine receptor CXCR4. This ligand-receptor complex plays im-

Correspondence to: Dr. Kazuko T Goto, Department of Dental Hygiene, Fukuoka College of Health Sciences, Fukuoka, 8140193, Japan; Tel: +81-92801-0411 ex4153; Fax: +81-92-801-4473; E-mail: kazuko@college.fdcnet.ac.jp portant roles in the development, leukocyte migration, and metastasis processes. SDF-1 reportedly promoted new bone formation upon topical administration in a rat skull defect model. It has also been recently reported that SDF-1 and bone morphogenetic protein-2 (BMP-2) induce bone formation ${ }^{9}$.

Here, we postulated that hyperocclusion MS induces the production of protective chemokines, including SDF-1, to maintain alveolar bone density and quality during occlusal trauma. Using in vivo and in vitro hyperocclusion models, we examined the effects of iMS on the relevance between chemokine expression in PDL and the maintenance of the bone marrow.

\section{Materials and Methods}

The protocol was reviewed and confirmed by the Research Ethics Committee (Approval No. 116). All animal experiments and follow the necessary procedures the guidelines established by the Animal Care Committee.

\section{Cell Culture}

Mouse PDL cells isolated from healthy mouse incisors and molars, as described previously ${ }^{10)}$. To avoid contamination by the gingivae and dental pulp, only the middle of each root was used. The cells were cultivated in a silicon-rubber chamber in $\alpha$-minimum essential medium containing $10 \%$ fetal bovine serum at $37^{\circ} \mathrm{C}$ in a humidified atmosphere with $5 \% \mathrm{CO}_{2}$. 


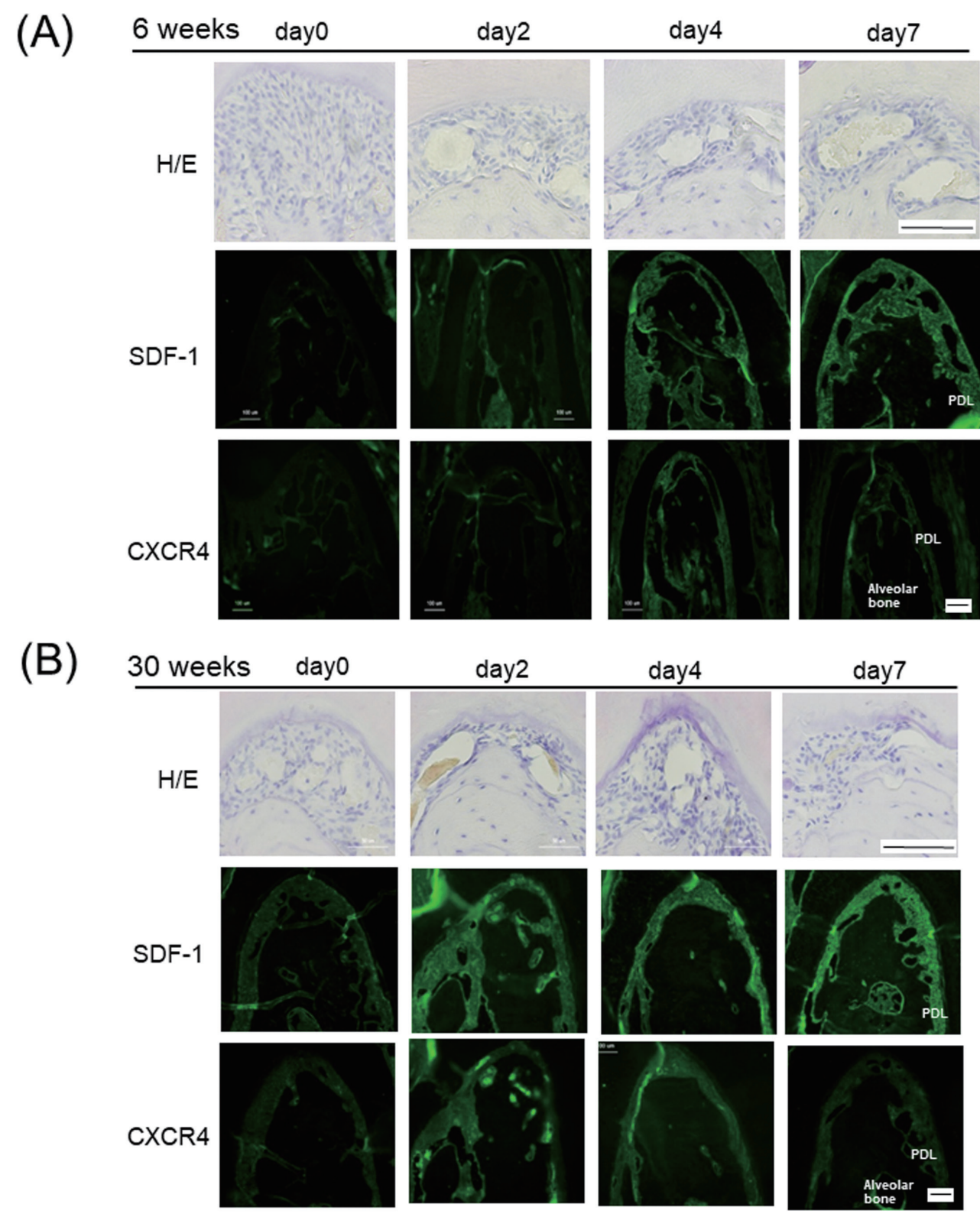

Figure 1. In vivo expression of stromal cell-derived factor-1 (SDF-1) and CXCR4 in PDL tissues, dental pulp, and bone marrow of hyperocclusion mouse models at 6 and 30 weeks. Hematoxylin-eosin (HE) staining and immunohistochemical staining of SDF-1 and CXCR4 in hyperocclusion mouse models at 6 weeks (A) and 30 weeks (B) are shown. Scale bars indicate $100 \mu \mathrm{m}$.

\section{In vitro Hyperocclusion Model}

Mouse PDL cells were cultivated in a $50-\mathrm{cm}^{2}$ silicon chamber at a density of $5 \times 10^{5}$ cells $/ \mathrm{cm}^{2}$. Attachments for silicon chamber was stretching apparatus (STB-140; Strex Co. Ltd., Osaka, Japan) and then subjected to intermittent uniaxial stretching for 1-7 days (iMS; conditions, $60 \mathrm{~s} /$ return; resting time, $29 \mathrm{~s}$; stretch length, $1.6 \mathrm{~mm}$; stretch ratio, $105 \%)$

\section{In vivo Hyperocclusion Model}

Experiments with animals were performed in accordance with the ethical guidelines for animal experiments established by Fukuoka Dental College (Approval No. 000233). Six- and 30- weeks of age ddY mice were used that purchased from Kyudo Animal Co, Ltd., Tosu, Japan. Anesthesia was induced by $2 \%$ isoflurane (Abbott Laboratories, Abbott
Park, IL, USA) and an air mixture gas flow of 11 / min using an anesthesia gas machine (Anesthesia machine SF-B01; MR Technology, Inc., Tsukuba, Ibaraki, Japan). To stimulate hyperocclusal MS, three maxillary molars in right side were attached a stainless steel wire using methyl-methacrylate resin (Super-Bond; Sun Medical Inc., Shiga, Japan). Stainless steel wire $(0.3 \mathrm{~mm}$ in diameter $)$ was glued to the occlusal surfaces of the right maxillary molars.

\section{Immunohistochemistry}

Tissue blocks were obtained from the mandible and soaked in $10 \%$ EDTA for $12 \mathrm{~h}$ at $4{ }^{\circ} \mathrm{C}$. After decalcification for 3 weeks at $4{ }^{\circ} \mathrm{C}$, the tissue blocks were washed overnight in phosphate-buffered saline containing $30 \%$ sucrose, buried in OCT compound, and frozen in dry ice/isopentane. Bone slices (thickness, $6 \mu \mathrm{m}$ ) were performed using a Frigocut 
(A)
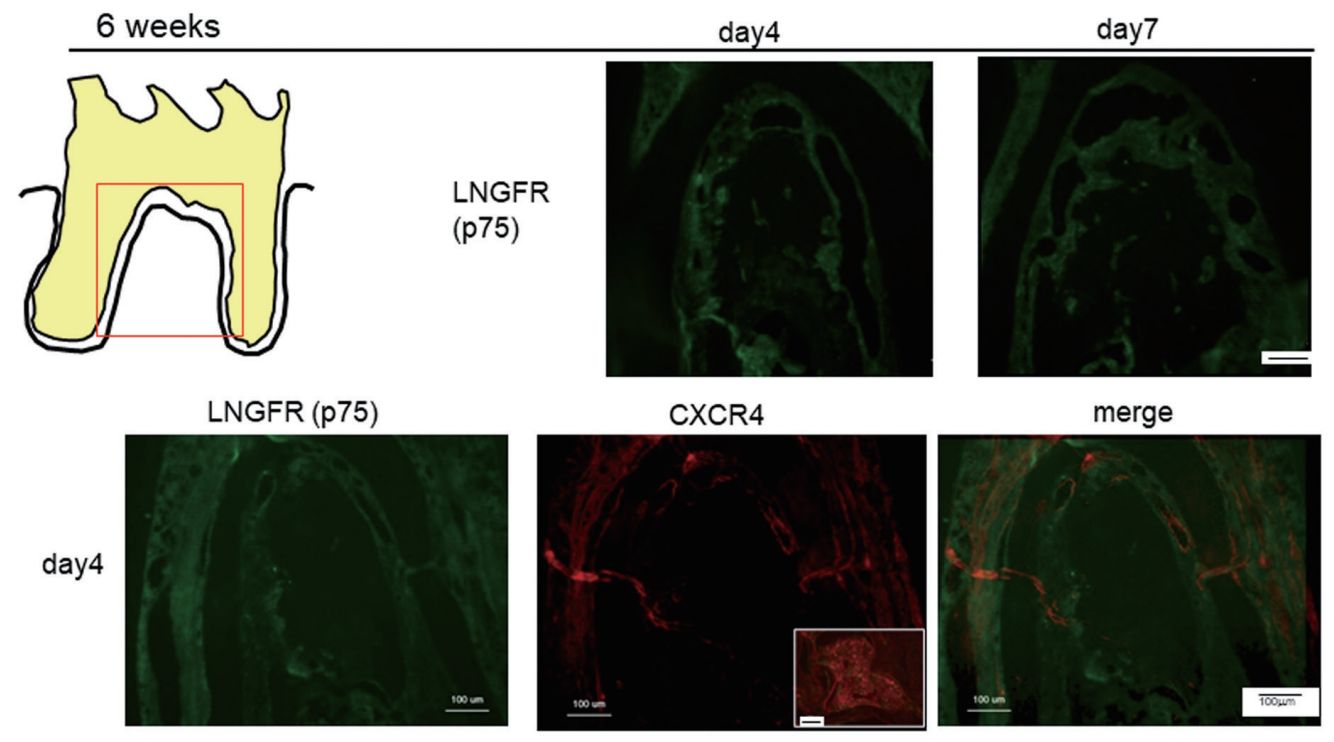

(B)
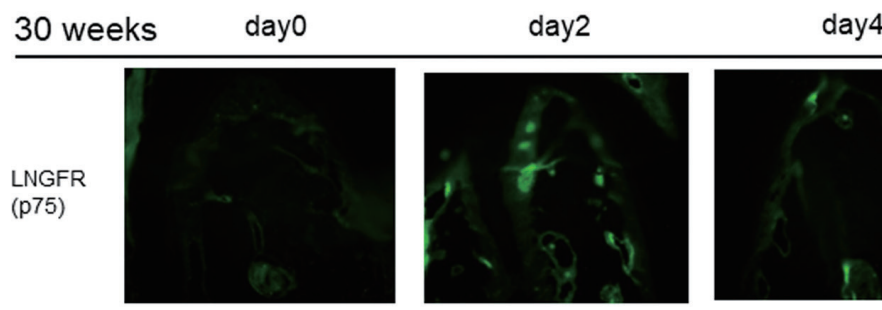

ay4

day7
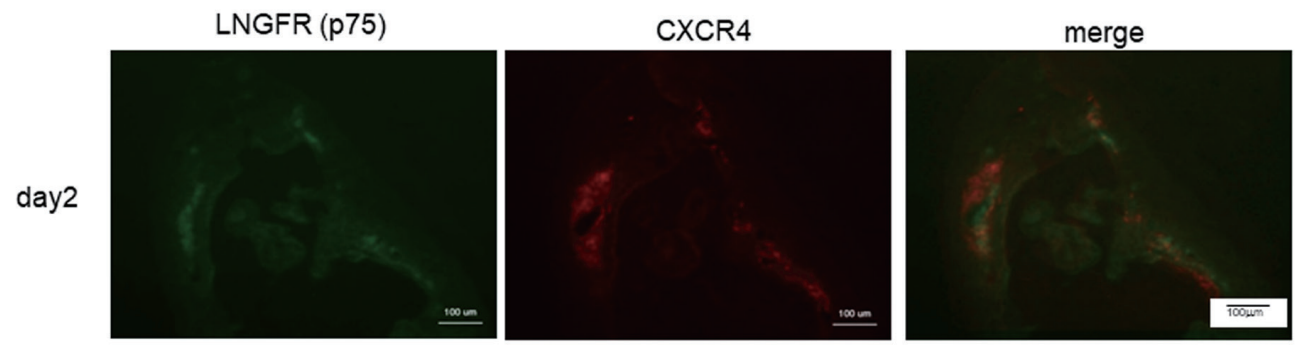

Figure 2. In vivo expression of LNGER (p75) and CXCR4 in the periodontal tissues of the hyperocclusion mouse models at 6 and 30 weeks. Immunohistochemical staining of LNGFR and CXCR4 in hyperocclusion mouse models at 6 weeks (A) and 30 weeks (B) is shown. Scale bars indicate $100 \mu \mathrm{m}$. Inlet in (A) is a low magnification image.

CM1850 (Leica, Wetzlar Germany). Slices were incubated with primary antibodies in a humidified chamber overnight at $4^{\circ} \mathrm{C}$. Antibodies were visible using an Alexa Fluor-conjugated IgG secondary antibody (1 mg/ $\mathrm{ml}$, Molecular Probes, OR, USA) after incubation for $30 \mathrm{~min}$ at room temperature. Nuclear staining was performed using 4,6-diamidino-2-phenylindole (Dojindo, Kumamoto, Japan).

\section{Western Blot Analysis}

Cells were dissolved in a lysis buffer $(\mathrm{pH}, 7.5)$ containing $20 \mathrm{mM}$ Tris- $\mathrm{HCl}, 200 \mathrm{mM} \mathrm{NaCl}, 1 \%$ Triton X-100, $1 \mathrm{mM}$ DTT, and protease inhibitors (Roche, Basel, Switzerland). The protein content of the samples was assayed using a protein assay kit (Pierce, Hercules, CA, USA), in accordance with the manufacturers' protocol. Protein samples $(20 \mu \mathrm{g})$ were subjected to $5 \%-20 \%$ sodium dodecyl sulfate-polyacrylamide gel electrophoresis and then electrophoretically transferred to a polyvinylidene fluoride membrane at $100 \mathrm{~V}$ for $1 \mathrm{~h}$ at $4^{\circ} \mathrm{C}$. The membrane was incubated overnight at $4^{\circ} \mathrm{C}$ with antibodies against the targeted chemok- ines at 1:500 dilutions in a 5\% dry skim milk solution. The blots were then incubated with a secondary antibody for $30 \mathrm{~min}$ at room temperature, followed by incubation for $1 \mathrm{~h}$ with horseradish peroxidase-conjugated goat anti-rabbit or mouse $\operatorname{IgG}$ diluted at 1:2,500 in 5\% dry skim milk-TTB and developed using an enhanced chemiluminescence system (GE, Arlington Heights, IL, USA).

\section{Statistical Analysis}

Data are expressed as the means and standard error of the number, $n$, of cells. Differences were assessed using paired one-way analysis of variance, as appropriate. $P<0.05$ was considered statistically significant.

\section{Results}

Intermittent MS in PDL cells transiently decreases and gradually increases SDF-1 gene expression

We have reported that iMS loading of human PDL cells for two days 
(A)

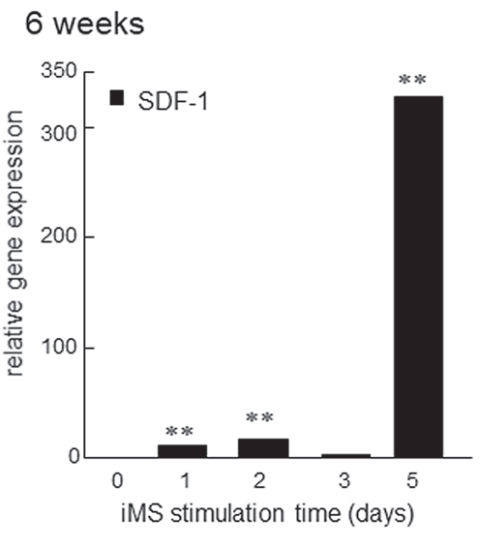

30 weeks

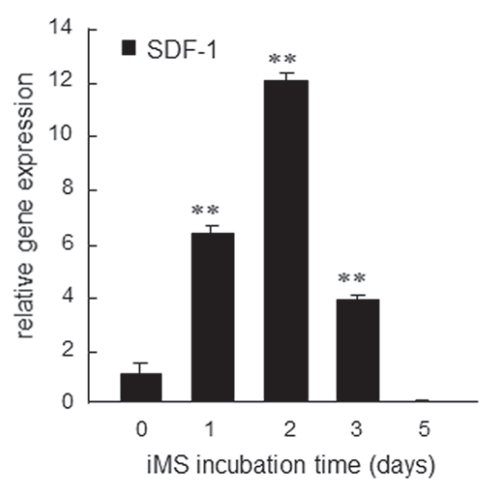

(C)
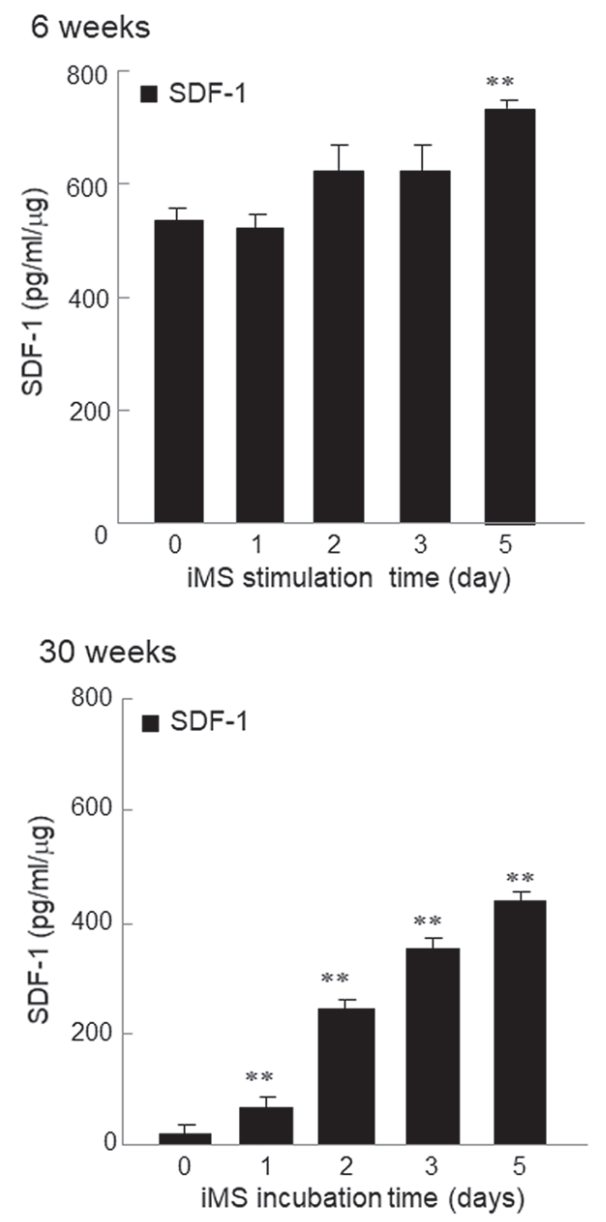

Figure 3. In vitro hyperocclusion models of the expressions of SDF-1 mRNA, protein, and secretion using hyperocclusion mouse models at 6 and 30 weeks. (A) qRT-PCR analysis of SDF-1 mRNA expression induced by intermittent mechanical stress (iMS) using the stretcher of a silicon chamber in hyperocclusion mouse models at 6 and 30 weeks. (B) Expression of iMS-induced SDF-1 protein in hyperocclusion mouse models at 6 and 30 weeks. (C) iMS induced secretion of SDF-1 protein in the hyperocclusion mouse model at 6 and 30 weeks.

upregulated the expression of the bone resorption associated cytokines and chemokines genes compared with unloaded cells in an in vitro model using DNA microarray analysis ${ }^{11}$. In contrast, iMS also downregulated the expression of SDF-1 gene, a self-renewal chemokine in stem cells of the bone marrow on day 2 after stimulation in human PDL cells.

We furthermore investigated the time course of iMS-induced SDF-1 expression using the in vivo hyperocclusion model of young (6 weeksold) and aged (30 weeks-old) mice. No significant inflammation was observed by hematoxylin-eosin staining until day 7 after MS loading (Fig. 1) in two types of young and aged mice. Although little change in the expression of SDF-1 was identified by immunohistochemical staining until day 2 following hyperocclusion MS loading, the expression of SDF-1 gradually increased in PDL tissues after day 4 in the in vivo hyperocclusion model with 6 weeks-old mice. In contrast, MS rapidly increased the expression of SDF-1 in PDL tissues on day 2 after MS in the hyperocclusion model with 30 weeks-old mice.

The hyperocclusional MS also increased the expression of CXCR4, SDF-1 receptor in the dental pulp and alveolar bone marrow on day 4 or 2 in the hyperocclusion models at 6 or 30 weeks-old mice, respectively.

\section{Co-localization of CXCR4 and LNGER (p75) in bone marrow}

To investigate the properties of MS-induced CXCR expression, we examined the expressions of mesenchymal cell marker proteins in the periodontal tissues in the hyperocclusion model mice. CXCR4 expression was almost co-localized in a mesenchymal cell marker, LNGER (p75)-positive cells in the dental pulp and bone marrow of the hyperocclusion mice model.

The co-expressions of LNGER (p75) and CXCR4 were increased on day 4 in the hyperocclusion model with 6 weeks-old mice (Fig. 2). In contrast, MS rapidly increased both the expression of LNGER (p75) and CXCR4 on day 2 after MS stimulation in PDL tissues of the hyperocclusion model with 30 weeks-old mice.

\section{Time-dependent expression of SDF-1 in PDL cells stimulated by iMS}

To disclose the acute response to MS, we used quantitative PCR, ELISA, and Western blots to examine changes in the expression of SDF-1 on days $0,1,2,3$, and 5 following MS loading in PDL cells. In the in vitro hyperocclusion model, iMS loading significantly upregulat- 
ed the expression of SDF-1 mRNA on day 5 after stimulation in PDL cells with 6 weeks-old mice (Fig. 3A). In contrast, MS quickly upregulated the expression of SDF-1 mRNA on day 2 after stimulation in PDL cells with 30 weeks-old mice. SDF-1 protein expression was slightly increased at day 3 after MS in PDL cells with 6 or 30 weeks-old mice (Fig. 3B). SDF-1 was also significantly produced at day 5 after iMS from PDL cells with 6 weeks-old mice (Fig. 3C). SDF-1 secretion was lower concentration in 30 weeks-old mice than that of 6 weeks-old mice day 0 before iMS. SDF-1 rapidly produced at day 2 after iMS from PDL cells with 30 weeks-old mice, but a few at 5 day after iMS in PDL cells with 6 weeks-old mice.

\section{Discussion}

We investigated the hyperocclusion MS-induced expression of chemokines associated to alveolar bone metabolism in PDL and their receptors in the bone marrow cells in in vitro with MS-treated PDL cells and in vivo models with traumatic hyperocclusion. The in vitro and in vivo models similarly revealed the following results during hyperocclusion, MS significantly upregulated the expression of SDF-1 in PDL tissue on 4-7 days in 6weeks-old mice or 2-7 days in 30 weeks-old mice after MS loading, indicating in quick protection to MS damages in PDL tissues in aged mice compared to that of young mice. The MS-induced SDF-1 upregulation may effective against stress and the initiation of osteoblasts differentiation from mesenchymal bone marrow cells, resulting in the maintenance of alveolar bone density and quality during occlusal traumatism. MS loading for $48 \mathrm{~h}$ has previously reported to lead to a transient decrease in the expression of MS-protective and osteogenic chemokines in human PDL cells using DNA microarrays. MS in present experiments particularly induced SDF-1 expression in PDL tissue of the in vivo mouse models with young and aged mice. We found that the time course of MS-induced SDF-1 expression is likely to dramatically increase following transiently decrease in mouse PDL tissues. Furthermore, both the expression and localization of SDF-1 in vivo were similar to those recognized in the in vitro models. The data indicated that CXCR4, a SDF-1 receptor also increased by MS in mesenchymal cells of alveolar bone marrow, resulting in protection of hyperocclusal damages in periodontal tissues. The SDF-1 has been known to be upregulated the MSCs in the portion of bone repair after femoral bone fracture ${ }^{12)}$. The circulating osteoblast progenitor cells in BMCs have reported to be recruited in bone formation via SDF-1/CXCR4 axis ${ }^{13)}$.

These findings suggested that the alveolar bone maintenance against MS induced the osteoblasts differentiation from mesenchymal bone marrow cells via upregulation of SDF-1 in damaged PDL tissues, resulting in the quick initiation in old mice compared to young mice occlusal traumatism.

MS loading is well known to induce receptor activator of nuclear factor kappa-B ligand (RANKL) expression in PDL both in vivo and in vitro $^{3,14)}$. Jung et al. reported a possible mechanism for localizing stem cells in the appearing bone marrow, whereby accrued expression of SDF-1 in the local bone marrow environment along with diminished SDF-1 in serum may create a homing gradient ${ }^{15)}$. The role provided that SDF-1 in BMP2-induced osteogenic differentiation of MSCs as a perturbing signal affects the differentiation of MSCs toward osteoblastic cells in response to BMP2 stimulation ${ }^{16}$. Together with our present data, these effects propose that SDF-1 is involved in alveolar bone metabolism via SDF-1/CXCR4 signaling during traumatic hyperocclusion.

It is known that SDF-1 expression is temporally and spatially associated with tooth eruption, arthritic bone destruction, osseous inflammation, and the developmental regulation of bone remodeling ${ }^{17-19)}$. Mesen- chymal stem cells reportedly express CXCR4 and CCR2 and reacted to chemokine ligands, such as SDF-1 and MCP- $1^{20)}$. The in vivo and in vitro experimental models used in the present study indicated that the excessive MS associated with hyperocclusion as well as the orthodontic model mice with retraction and compressed stress resulted in the migration of SDF-1 in PDL tissues and its receptor CXCR4 in the bone marrow, including in the mesenchymal stem cells. These results demonstrate that SDF-1 plays not only an MS-dependent role in bone resorption but an MS-protective role in bone formation during alveolar bone remodeling.

Both SDF-1 mRNA and protein reportedly increase during bone graft repair, and an anti-SDF-1 antibody reportedly inhibits new bone formation $^{21}$. SDF-1 expression was increased around defects at already 1 week after injury, and BMCs were mobilized to the defects ${ }^{22}$. These results suggest that SDF-1 may regulate and thus promote alveolar bone formation during occlusal traumatism.

Furthermore, the level of $\mathrm{PGE}_{2}$ and IL- $1 \beta$ secreted from elder PDL tissues has been reported to be higher than that of young PDL tissues ${ }^{23}$. Our reported also indicated the excess MS during hyperocclusion protectively increased the expression of collagen type XII in aged mice compared to that of younger mice ${ }^{24)}$.

These finding, together with our data suggested that excess MS to elder periodontal tissues quickly may defend and maintain their present characteristics and shape compare to that of younger.

In conclusion, we demonstrated that MS induces SDF-1 secretion from PDL tissues. SDF-1 binds to its receptor CXCR4 in mesenchymal stem cells in the bone marrow and might perform an important role in the maintenance of alveolar bone metabolism with inducing osteoblast differentiation.

\section{Acknowledgments}

This work was supported by Grants-in-Aid for Scientific Research from the Ministry of Education, Culture, Sports, Science and Technology of Japan (15K11187 and 19K10234) and the Private University Research Branding Project at Fukuoka Dental College and Fukuoka College of Health Sciences. We thank M. Arico from Edanz Group (www. edanzediting.com/ac) for editing a draft of this manuscript.

\section{Conflict of Interest}

The authors have no conflict of interest to declare.

\section{References}

1. Field C, Li Q, Li W and Swain M. Influence of tooth removal on mandibular bone response to mastication. Arch Oral Biol 53: 11291137,2008

2. Gerard C and Rollins BJ. Chemokines and disease. Nat Immunol 2: 108-115, 2001

3. Goto KT, Kajiya H, Nemoto T, Tsutsumi T, Tsuzuki T, Sato H and Okabe K. Hyperocclusion stimulates osteoclastogenesis via CCL2 expression. J Dent Res 90: 793-798, 2011

4. Graves DT, Jiang Y and Valente AJ. Regulated expression of MCP1 by osteoblastic cells in vitro and in vivo. Histol Histopathol 14: 1347-1354, 1999

5. Herberg S, Aguilar-Perez A, Howie RN, Kondrikova G, Periyasamy-Thandavan S, Elsalanty ME, Shi X, Hill WD and Cray JJ. Mesenchymal stem cell expression of SDF-1 $\beta$ synergizes with BMP-2 to augment cell-mediated healing of critical-sized mouse calvarial defects. J Tissue Eng Regen Med 11: 1806-1819, 2017

6. Hosogane N, Huang Z, Rawlins BA, Liu X, Boachie-Adjei O, Bos- 
key AL and Zhu W. Stromal derived factor-1 regulates bone morphogenetic protein 2-induced osteogenic differentiation of primary mesenchymal stem cells. Int J Biochem Cell Biol 42: 1132-1141, 2010

7. Jung Y, Wang J, Schneider A, Sun YX, Koh-Paige AJ, Osman NI, McCauley Lk and Taichman RS. Regulation of SDF-1 (CXCL12) production by osteoblasts; a possible mechanism for stem cell homing. Bone 38: 497-508, 2006

8. Kim MS, Magno CL, Day CJ and Morrison NA. Induction of chemokines and chemokine receptors CCR2b and CCR4 in authentic human osteoclasts differentiated with RANKL and osteoclast like cells differentiated by MCP-1 and RANTES. J Cell Biochem 97: 512-518, 2006

9. Kimura Y, Komaki M, Iwasaki K, Sata M, Izumi Y and Morita I. Recruitment of bone marrow-derived cells to periodontal tissue defects. Front Cell Dev Bioll 19: 1-6, 2014

10. Kitaori T, Ito H, Schwarz EM, Tsutsumi R, Yoshitomi H, Oishi S, Nakano M, Fujii N, Nagasawa T and Nakamura T. Stromal cell-derived factor 1/CXCR4 signaling is critical for the recruitment of mesenchymal stem cells to the fracture site during skeletal repair in a mouse model. Arthritis Rheum 60: 813-823, 2009

11. Rahimi P, Wang CY, Stashenko P, Lee SK, Lorenzo JA and Graves DT. Monocyte chemoattractant protein-1 expression and monocyte recruitment in osseous inflammation in the mouse. Endocrinology 136: 2752-2759, 1995

12. Okada K, Kawao N, Yano M, Tamura Y, Kurashimo S, Okumoto K, Kojima K and Kaji H. Stromal cell-derived factor-1 mediates changes of bone marrow stem cells during the bone repair process. Am J Physiol Endocrinol Metab 1: 310: 15-23, 2016

13. Otsuru S, Tamai K, Yamazaki T, Yoshikawa H and Kaneda Y. Circulating bone marrow-derived osteoblast progenitor cells are recruited to the bone-forming site by the CXCR4/stromal cell-derived factor-1 pathway. Stem Cells 26: 223-234, 2008

14. Sasaguri K, Jiang H and Chen J. The effect of altered functional forces on the expression of bone-matrix proteins in developing mouse mandibular condyle. Arch Oral Bio 43: 83-92, 1998

15. Tsutsumi T, Kajiya H, Goto KT, Takahashi Y and Okabe K. Hyperocclusion up-regulates CCL3 expression in CCL2- and CCR2-deficient mice. J Dent Res 92: 65-70, 2013

16. Verna C, Melsen B and Melsen F. Differences in static cortical bone remodeling parameters in human mandible and iliac crest. Bone 25: 577-583, 1999

17. Volejnikova S, Laskari M, Marks SC Jr and Graves DT. Monocyte recruitment and expression of monocyte chemoattractant protein-1 are developmentally regulated in remodeling bone in the mouse. Am J Pathol 150: 1711-1721, 1997

18. Walker CG, Ito Y, Dangaria S, Luan X and Diekwisch TGH. RANKL, osteopontin, and osteoclast homeostasis in a hyperocclusion mouse model. Eur J Oral Sci 116: 312-318, 2008

19. Wei H, Zhao X, Yuan R, Dai X, Li Y and Liu L. Effects of PB-EPCs on homing ability of rabbit BMSCs via endogenous SDF-1 and MCP-1. PLOS One 2015: doi.org/ 10.1371/journal.pone.0145044.

20. Yoshinaga Y, Ukai T, Abe Y and Hara Y. Expression of receptor activator of nuclear factor kappa B ligand relates to inflammatory bone resorption, with or without occlusal trauma, in rats. J Periodontal Res 42: 402-409, 2007

21. Zlotnik A and Yoshie O. Chemokines: a new classification system and their role in immunity. Immunity 12: 121-127, 2000

22. Zlotnik A, Yoshie O and Nomiyama H. The chemokine and chemokine receptor superfamilies and their molecular evolution. Genome Biol 7: 243, 2006

23. Shimizu N, Yamaguchi M, Uesu K, Goseki T and Abiko Y. Stimulation of prostaglandin E2 and interleukin-1beta production from old rat periodontal ligament cells subjected to mechanical stress. J Gerontol A Biol Sci Med Sci 55: 489-495, 2000

24. Tsuzuki T, Kajiya H, Goto KT, Tsutsumi T, Kawaguchi T and Okabe $\mathrm{K}$. Effect of aging on historogical and functional changes by hyperocclusion in mouse periodontal ligament. J Fukuoka Dent Coll 44: 119-127, 2019 